\title{
Development of Discoid Products Stacking Machine Based on PLC
}

\author{
Wenguang Zheng ${ }^{\mathrm{a}}$, Zening $\mathrm{Xu}^{\mathrm{b}}$ and Xiaoguang $\mathrm{Yu}^{\mathrm{c}}$
}

School of Mechanical Engineering and Automation, University of Science and Technology Liaoning Anshan, China, 114051

azhengwg2005@163.com, buuzening-1@163.com, cyuxiaoguang58@163.com

Keywords: Discoid products, Stacking, Mechatronics, PLC

\begin{abstract}
Binding the actual production of one sporting goods factory, in order to meet the needs of automatic production line of shooting flying saucer, the discoid products stacking machine was developed based on PLC, carried out mechanical system design of discoid products stacking machine and control system development based on PLC. After production testing proved reasonable structure of mechatronics systems, easy to operate, reliable, able to meet the requirements of automated production, with a strong practical and innovative, have some application value.
\end{abstract}

\section{Introduction}

In order to meet the need of the production line for skeet shooting in a factory, the stacking mechanism system of discoid products is designed, the controlling system based on PLC is improved, and the stacking for discoid products is developed. The stacker for discoid products is designed for packing, one of the all processes, by using mechatronics system, which can ensure the continuity and increase the producing speed. The production line synchronizing functions are realized by PLC controlling the motor speed. According to the condition of the equipment of the operation, the actual meaning is obvious.

\section{Design of the mechanism system}

Stacking is the last operation of the production line. The design is adopts the cylinder and sensor and take advantage of the counting function of the PLC. The products are divided into four lines in the production line. The stacker work automatically, when which reached a certain level, pushing cylinder put one line products forward which are packed workers. Because the lower speed of the workers, the accumulation exists, which in order to deal with the Storing device is needed. The design above can heighten produheighten productivityctivity.

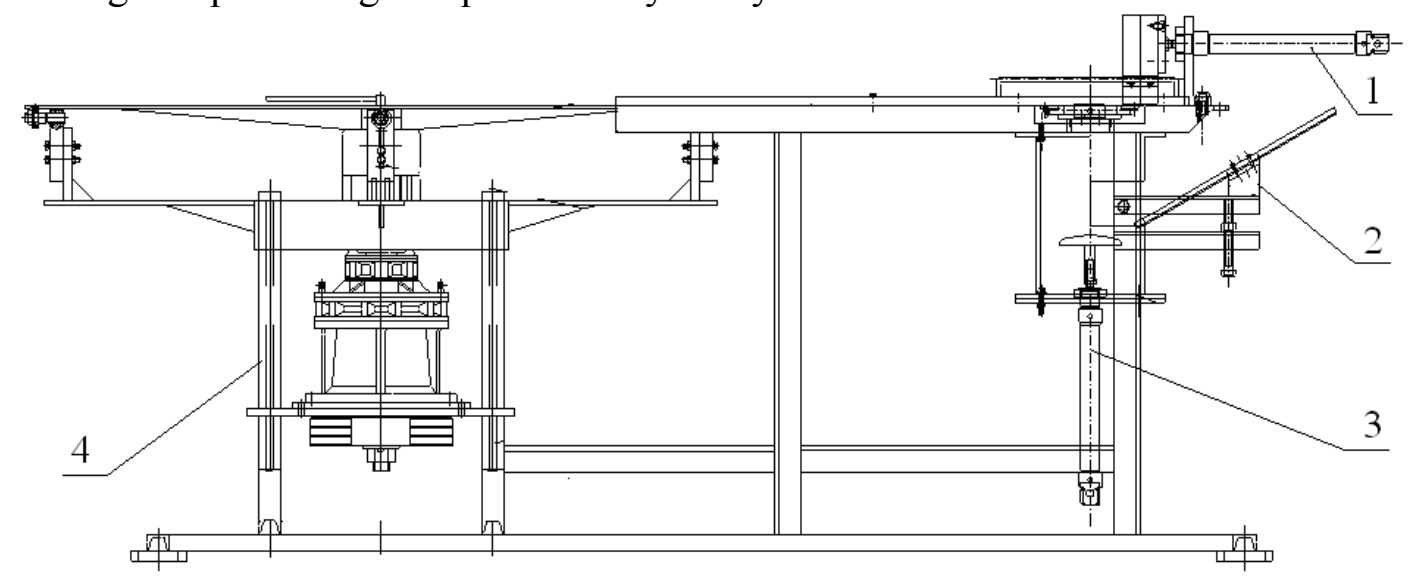

1. Pushing device 2. Transportation fixing device 3. Stacking device 4. Storing device

Fig. 1. Blueprint of discoid products stacking system 
Transportation fixing device. Transportation fixing device is consisted of a guide frame and four transportation fixing platforms. Guide frame is connected to stacker with bolts. The front-end of the transportation fixing device is installed at the end of the conveyer belt, which lead the products to the fixing platform. The beck-end of the transportation fixing device is connected to the stacking device, which lead the products to the stacking device, to make sure the stacking of the products. The input fixing device of the products divides the products into lines and put them into the scheduled position, which can make the products slip naturally to the tray of the stacking, the tray lift the products, and the process of stack is completed. In order to be fixed and adjusted freely, the frame foundation of the transportation fixing device adapt adjustable form, which can adjust the angle of the position to make the products go into the stacking device fluently.

Stacking device. The principle of the stacking device is that a tray is fixed on a cylinder on end. The tray is shaped like a mushroom, and the outline is identical with the inner glassy surface of the products, has smooth surface, and can be fixed accurately. When the cylinder moves upwards to finish the process of stacking, the first products is put on a spring plate, and then as the second one is push forward, the first one is jacked up, thus the two products were on the spring plate. With the circulation continuing, a series of products were on it. The number of the products is set, so after the number is reached, the pushing device continues.

Pushing device. Cylinder is still the power of the Pushing device. The cylinder is installed horizontally as usual, so it can move horizontally during the working process. A rubber pushing block, which will not injure the products, is installed at the front-end of the cylinder. When the number of the stacking reach a fixed level, the pushing cylinder moves forward to push the products onto the storing device.

Storing device. The storing device is made up of a motor, a reducer and a very large circle plate. The circle plate is placed on the same level as the storing device. The storing plate is made into internal arc surface which has the same diameter with the storing turn plate of the stacker, so that the two plates can be connected. In the working process, the storing plate moves in even circling motion according to the speed of the speed of the production line. When the workers' packing speed is lower, the accumulated products were pushed onto the storing plate immediately. Once the pushing cylinder moves, and one stack products was pushed onto the storing turn plate. An adjustment unit is designed to control the products placed circle by circle from the center to the edge of the turn plate, when a large number of products left can not be packed in time.

\section{Development of control system}

The principle of stacker. The system structure of the stacker is shown as fig.2. PLC controlling system consists of the transducer and PLC. PLC has the network interface to communicate with the upper machine and thus the production of the whole is realized.

When the system works, the speed of rotation can be adjusted freely by the PLC controlling system until the actual working need is satisfied. A functional relation between the cylinder parameter and stacking pushing parameter is established. The aim is to finish the task smoothly. every parameter of the PLC is used to control the production process and thus the quality of the production can be ensured. The transducer gives a signal by testing the conveying condition of the products on the conveyor belt to make the time when the products slip down to the tray delayed. And then a signal is sent to the stacking cylinder which begins to work to pile up the products. The working condition of the pushing cylinder is up to the stacking cylinder, which is controlled by the counter of PLC controlling system. The counter takes count of the number of the movement of the stacking cylinder, each of which is called a time of stacking. The adjustment of the storing turn plate generator is realized through the communication between the PLC and the transducer so that the production process is completed. 


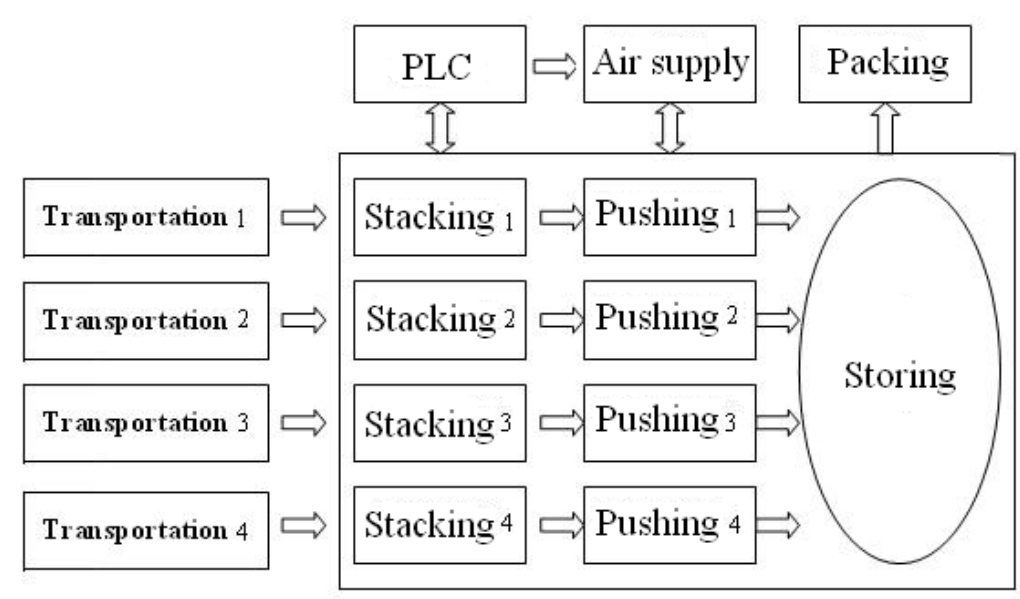

Fig. 2. The structure of discoid products stacking system

By testing the signals from PLC to transducer, the work site condition is compared with the preset parameter, to control the working state of the cylinder, and thus the steadier production can come true and the production efficiency can be raised.

Hardware composition. The hypogyny computer is S7-200 system of Siemens, and the central processing unit CPU226, the analogue output module EM232.

Program design. The process of the design of the main programmer is as fig.3 (A). Under the conditions for working, the main programmer works, through which the detailed controlling is made by using subprograms. The motor controlling function of the generator is shown as fig.3 (B). The flow chart of stacking System controlling function is shown as fig.4.
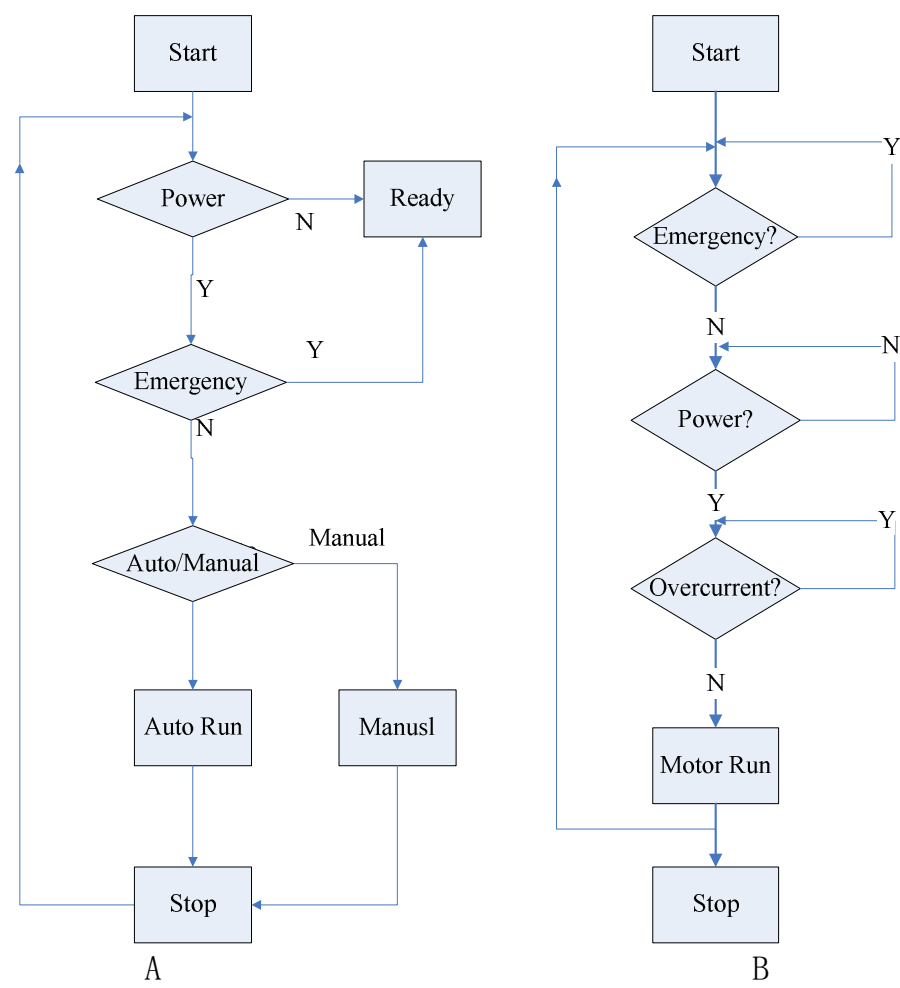

Fig. 3. Flow Chart of main program and motor control

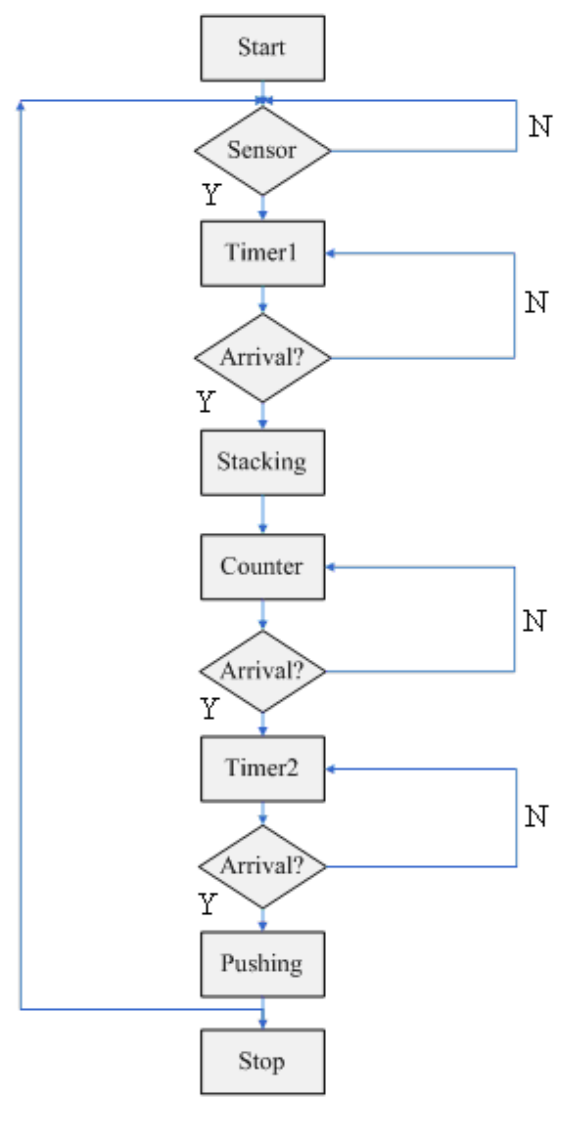

Fig. 4. Flow Chart of stacking System 
According to the technological requirements in worksite and the characters of the program software Step7 MicroWIN, the controlling process of the stacking system is programmed by the way of using main function. Main function is operated by the way of scan-round, which divided the production line into several parts, based on which functions is programmed. In main function, under the condition of operation conditions, the subprograms are invoked. According to the flow chart of stacker control system, the stacking process can be programmed with Step7 MicroWIN. Step7 MicroWIN programming software is used as the system development tools. With modular programming method, those subroutine such as logic control, delaying start-up and stop, speed closed-loop control, pneumatic system control are developed according to different requirements of each process. Discoid products stacking mechantronics system is an important link in the whole automatic production line of shooting flying saucer, so this invention has objective social and economic benefits.

\section{Conclusion}

With the technical requirement of the worksite of a factory, the principle of mechatronics systems is used to design the stacker for the products in the shape of plate, and the controlling system of discoid products stacker based on S7-200 is developed, so the automatic control comes true. The commissioning operation shows that discoid products stacker based on PLC is designed reasonably, is operated conveniently and runs safely and reliably. The discoid products stacking machine on PLC improves the work efficiency and reduces the labor intensity of the workers. The discoid products stacking machine on PLC meets the designing requirement. Its application makes great economic result, and is very innovative and practical.

\section{References}

[1] Ma Dongyuan. An Introduction of Automatic Palletizing Equipment Used in Membrane Production Lines. China Building Waterproofing, J. 2008.12:13-15.

[2] Geng Zhiyuan. Development of The Dish Automatic Stacking System, D. University of Science and Technology Liaoning. 2007.

[3] Xia Min. Stacking and Counting of Tobacco Bales, J. Tobacco Science \& Technology. 2005.7(216):46-48.

[4] Liu Yongxian. Research on the Piled Machine of the 2.2M Corrugated Carton Production Line, D. Northeastern University. 2010.

[5] $\mathrm{Hu}$ Hongguo, Gao Jianhua, Yang Ruqing. The development of palletizing technology, J. Modular Machine Tool \& Automatic Manufacturing Technique, 2000.6:7-9.

[6] Zheng Wen-guang, Xu Ze-ning, Hu Su-ying. Research and application of packing steel strips rewinding with PLC [J]. Journal of University of Science and Technology Liaoning, 2009(2):143- 147. 